\title{
Synthesis and Optical Properties of Anisotropic Metal Nanoparticles
}

\author{
Encai Hao, ${ }^{1}$ George C. Schatz,,${ }^{1,2}$ and Joseph T. Hupp ${ }^{1}$
}

Received January 15, 2004; revised March 24, 2004; accepted March 24, 2004

\begin{abstract}
In this paper we overview our recent studies of anisotropic noble metal (e.g. gold and silver) nanoparticles, in which a combination of theory and experiment has been used to elucidate the extinction spectra of the particles, as well as information related to their surface enhanced Raman spectroscopy. We used wet-chemical methods to generate several structurally well-defined nanostructures other than solid spheres, including silver nanodisks and triangular nanoprisms, and gold nanoshells and multipods. When solid spheres are transformed into one of these shapes, the surface plasmon resonances in these particles are strongly affected, typically red-shifting and even splitting into distinctive dipole and quadrupole plasmon modes. In parallel, we have developed computational electrodynamics methods based on the discrete dipole approximation (DDA) method to determine the origins of these intriguing optical features. This has resulted in considerable insight concerning the variation of plasmon wavelength with nanoparticle size, shape and dielectric environment, as well as the use of these particles for optical sensing applications.
\end{abstract}

KEY WORDS: Anisotropic metal nanoparticles; shape-dependent properties; discrete dipole approximation; surface plasmon resonances; electric field.

\section{INTRODUCTION}

Nanoscience and nanotechnology is an emerging and rapidly developing field that broadly encompasses the fundamental understanding and resulting technological advances arising from the exploitation of materials having at least one dimension at the nanometer length scale. The remarkable size-, surface-, and shape-dependence of physical, optical, and electronic properties of nanoscale materials make them compelling components of modern materials chemistry [1-6]. During the last decade, significant progress has been achieved in controlling the sizes of materials [7], and engineering their surfaces [2,3,8-10].

More recently, there have been important discoveries concerning the generation of anisotropic nanoparticles with well-defined structures other than solid spheres.

\footnotetext{
${ }^{1}$ Department of Chemistry, Northwestern University, Evanston, Illinois 60208-3113.

${ }^{2}$ To whom correspondence should be addressed.E-mail: schatz@chem. northwestern.edu
}

These include nanoscale rods [11-18], disks [19-23], triangular prisms [4,24-28], multipods [29-32], cubes [33,34], and nanoshells [35-40]. Beyond their aesthetic appeal, these new structures are compelling for many fundamental and practical technological reasons. First, their synthesis has motivated experimental progress in understanding the intrinsic shape-dependent properties of metal and semiconductor nanocrystals $[4,6,18,20,28,41,42]$. Second, some of these structures feature optical and electrical properties that make them desirable for emerging applications entailing bio-labels, photovoltaic behavior [43], chemical sensing [44], and surface enhanced Raman scattering (SERS) [5,45,46], among others. Third, the particle growth mechanism is amenable to study in some cases, which in turn makes possible the prediction and systematic manipulation of the final nanocrystal structure $[17,30]$. Fourth, these nanoparticles provide new template materials for the generation of different nanostructures [47-49].

Accordingly, their synthesis also motivates the development and innovation of theoretical methods for describing the unique properties of these anisotropic 
nanoparticles [5]. Recently, a number of theoretical approaches have been developed, including the discrete dipole approximation (DDA) [5,50-52], finite difference time domain methods (FDTD) [53], and the modified long wavelength approximation (MLWA) [5,50]. Among them, the DDA is a particularly useful technique for describing isolated nanoparticles with arbitrary shape and a complex surrounding environment $[5,51]$. In the DDA, the object of interest is represented as a cubic array of $N$ polarizable elements. The response of this array to an applied electromagnetic field is then described by self-consistently determining the induced dipole moment in each element. This information can be used to determine far-field properties like extinction efficiencies and also near-field properties especially the electromagnetic field (E-field) near the particle surface.

Among the known anisotropic nanoparticles, nanorods are the most common as this structural motif is found in a broad range of materials, including $\mathrm{CdE}(\mathrm{E}=$ $\mathrm{Se}, \mathrm{Te})[16,42], \mathrm{Ag}[6,14], \mathrm{Au}[6,12,15,18], \mathrm{TiO}_{2}$ [13,54], and others [55]. Rod-shaped particles provide a useful way to control nanoparticle optical properties, as spherical $\mathrm{Ag}$ and Au nanoparticles have strong surface plasmon bands around 400 and $520 \mathrm{~nm}$, respectively, while $\mathrm{Ag}$ and $\mathrm{Au}$ nanorods usually show a red-shifted long-axis resonance and a slightly blue-shifted short-axis resonance [6]. The long-axis resonance wavelength strongly depends on the aspect ratio of the nanorods. Another important finding is that Au nanorods have a one million times stronger fluorescence yield than that of the spherical nanoparticles.

Recently it has been discovered that non-rod shaped anisotropic particles can also have interesting optical properties. For example, a photo-driven synthesis of Ag triangular nanoprisms has been developed in which the size of the particles is controlled by the wavelength of the photolysis. Interestingly, these Ag triangular nanoprisms exhibit intriguing optical features with distinctive electric dipole and electric quadrupole modes $[4,28]$.

Although some success has been achieved in developing synthetic methods for producing anisotropic nanoparticles, there are still great challenges to developing these methods, to understanding the shape-guiding mechanisms, and to the functional integration of these materials into real-world applicable devices. Most recently, a combination of experimental and theoretical results designed to elucidate the optical properties of anisotropic metal nanoparticles has been developed in our lab $[20,28,32,46,56]$. Wet-chemical methods have been used to prepare these materials, including Ag nanodisks, Ag prisms, "branched" Au nanocrystals, and Au nanoshells.

In this paper, we give an overview of the synthesis and characterization of gold and silver anisotropic nanoparti- cles that we have developed as well as the analysis of their optical properties using computational electrodynamics methods. Our goal will be to show both common elements in their synthesis as well as the diverse strategies that can be employed. In addition, our analysis of optical properties will emphasize sensitivity of the plasmon modes to particle structure, and the contrasting behavior of silver and gold particles. Although most of our analysis will be concerned with particles that we have synthesized, some aspects of related work, such as the silver triangle synthesis, will be included when useful.

\section{MATERIALS SYNTHESIS}

Although the synthetic methods used to make the anisotropic nanoparticles have been described previously $[20,32,56]$, here we give a brief summary so that some of the essential elements are described, as this will help in our later discussion of the particle structural and optical properties.

\section{Ag Nanodisks}

For a typical synthesis of the silver nanodisks [20], a fresh $\mathrm{Ag}$ solution containing $10 \%$ wt $\mathrm{AgNO}_{3}$ and 6\% ammonium hydroxide was prepared. $160 \mu \mathrm{L}$ of $110 \mathrm{~nm}$ diameter polystyrene (PS) spheres and $75 \mu \mathrm{L}$ of the $\mathrm{Ag}$ solution were added to $60 \mathrm{~mL}$ of somewhat aged $N, N$ dimethylformamide. Then, the solution was heated with stirring on a hotplate. Within 8-12 min, the solution color changed from yellow through red to purple, after which the temperature was quickly decreased via a cold-water bath. The product was purified by gradient centrifugation and then kept at $4^{\circ} \mathrm{C}$.

\section{Au "Multipods"}

In the synthesis of the Au "multipod" structures [32], $4 \mathrm{mg}$ of bis( $p$-sulfonatophenyl) phenylphosphine dihydrate dipotassium (BSPP) and $0.2 \mathrm{~mL}$ of $30 \% \mathrm{H}_{2} \mathrm{O}_{2}$ were added to $100 \mathrm{~mL}$ of $6.8 \times 10^{-3} \mathrm{M}$ sodium citrate solution. Under constant shaking, $200 \mu \mathrm{L}$ of $0.05 \mathrm{M} \mathrm{HAuCl}_{4}$ was added slowly at room temperature. Over several minutes, the solution color changed from colorless to blue. The resulting blue colloids were then kept in a refrigerator.

\section{Au Nanoshells}

Au nanoshells [56] have been prepared by reacting $\mathrm{HAuCl}_{4}$ with silver nanoparticles similar to the method 
published by Sun and Xia [44]. Briefly, Ag nanoparticles were prepared by injection of $\mathrm{NaBH}_{4}(50 \mathrm{mM}, 2 \mathrm{~mL})$ to an aqueous solution of $\mathrm{AgNO}_{3}(0.2 \mathrm{mM}, 100 \mathrm{~mL})$ in the presence of sodium citrate $(0.5 \mathrm{mM})$. The resulting $\mathrm{Ag}$ colloid was then kept at $70^{\circ} \mathrm{C}$ for $2 \mathrm{hr}$. While stirring, 0.68 $\mathrm{mL}$ of $\mathrm{HAuCl}_{4}(0.1 \mathrm{M})$ was added to $100 \mathrm{~mL}$ of the $\mathrm{Ag}$ colloid solution. A color change from yellow to red to dark blue was observed during the course of the replacement reaction. After $1 \mathrm{hr}$ of stirring, the particles were purified by gradient centrifugation, washed twice using an aqueous solution of sodium citrate $(0.3 \mathrm{mM})$, redispersed in $5 \mathrm{~mL}$ of sodium citrate $(0.3 \mathrm{mM})$, and finally kept at $4{ }^{\circ} \mathrm{C}$.

\section{Discrete Dipole Approximation (DDA) Calculations}

The DDA method provides a convenient method for describing light scattering from nanoparticles of arbitrary shape. Details of this method have been given elsewhere $[5,27,46,51,52,57,58]$, so here we summarize the essential elements. In this method the particle is represented as a grid of cubical elements. The induced dipole polarization $P_{i}$ in each element is determined from

$$
\boldsymbol{P}_{i}=\alpha_{i} \cdot \boldsymbol{E}_{\mathrm{loc}}\left(\boldsymbol{r}_{i}\right)
$$

where $\alpha_{i}$ and $\boldsymbol{r}_{i}$ are the polarizability and location of the $i$ th dipole, and $E_{\text {loc }}$ is the local electric field. The local field at each dipole is given by

$$
\boldsymbol{E}_{\mathrm{loc}, i}=\boldsymbol{E}_{0} \exp \left(\boldsymbol{i k} \cdot \boldsymbol{r}_{i}\right)-\sum_{j \neq i} \boldsymbol{A}_{i j} \cdot \boldsymbol{P}_{j}
$$

where $\boldsymbol{E}_{0}$ and $\boldsymbol{k}$ are the amplitude and wave vector of the incident field, respectively. The interaction matrix $\boldsymbol{A}$ has the form

$$
\begin{aligned}
\boldsymbol{A}_{i j} \cdot \boldsymbol{P}_{j}= & \frac{\exp \left(i k r_{i j}\right)}{\boldsymbol{r}_{i j}^{3}}\left\{k^{2} \boldsymbol{r}_{i j} \times\left(\boldsymbol{r}_{i j} \times \boldsymbol{P}_{j}\right)\right. \\
& \left.+\frac{\left(1-i k r_{i j}\right)}{\boldsymbol{r}_{i j}^{2}} \times\left[\boldsymbol{r}_{i j}^{2} \boldsymbol{P}_{j}-3 \boldsymbol{r}_{i j}\left(\boldsymbol{r}_{i j} \cdot \boldsymbol{P}_{j}\right)\right]\right\}_{(j \neq i)}
\end{aligned}
$$

where $k=\omega / c$. Substituting Eqs. 2 and 3 into 1, we can we generate the system of equations

$$
\boldsymbol{A}^{\prime} \cdot \boldsymbol{P}=\boldsymbol{E},
$$

where the off diagonal elements of the matrix, $A_{i j}^{\prime}$ are same as $A_{i j}$, and the diagonal elements of the matrix, $A_{i i}^{\prime}$, are $\alpha^{-1}$.

For a system with $N$ total dipoles, $\boldsymbol{E}$ and $\boldsymbol{P}$ are $3 N$ dimensional vectors, and $A^{\prime}$ is a $3 N \times 3 N$ matrix. Solving this set of $3 N$ complex linear equations, the polarizations $P$ are determined, and from this we determine the extinction cross-section $\left(C_{\text {ext }}\right)$ using

$$
C_{\text {ext }}=\frac{4 \pi k}{\left|E_{0}\right|^{2}} \sum_{i=1}^{N} \operatorname{Im}\left(\boldsymbol{E}_{\mathrm{inc}, i}^{*} \cdot \mathbf{P}_{i}\right)
$$

All calculations here refer to water as the external dielectric for these novel materials. The dielectric constant in all calculations has been taken from Palik [59] but smoothed as described by Jensen et al. [51]

\section{RESULTS AND DISCUSSION}

\section{Ag Triangular Nanoprisms}

Before examining the optical properties of the particles described above, we first consider the properties of $\mathrm{Ag}$ triangular nanoprisms that were synthesized by Mirkin and coworkers [4,28]. These particles are relatively monodisperse, with edge lengths in the 30-120 nm range and heights of about $15 \mathrm{~nm}$. Their synthesis starts with a standard reduction of $\mathrm{AgNO}_{3}$ to produce small spherical particles, and then these are converted to the triangular prisms by visible light illumination in the presence of the same BSPP surfactant that we used to make nanoscale $\mathrm{Au}$ multipods. These particles have a plasmon resonance spectrum that is shown in Fig. 1 for edge lengths of 30, 60,100 and $150 \mathrm{~nm}$. These results are from DDA calculations, but they are in excellent agreement with experiment $[4,28]$ so we don't present the latter. We see in this figure that for all particles there are three dominant peaks in the extinction spectrum, corresponding to different modes of plasmon excitation. From the DDA calculations, we find that the red-most band, which is the most intense, corresponds to in-plane dipolar excitation. Also, the middle resonance is associated with in-plane quadrupole excitation and the bluest resonance, which is always at 335 $\mathrm{nm}$, refers to out-of-plane quadrupole excitation. For the largest particles, additional peaks are found that involve high multipolar excitation.

We see from Fig. 1 that the calculated extinction spectrum is very sensitive to its edge length, with the in-plane dipole and quadruple wavelengths increasing linearly with increasing edge length. Further calculations indicate that the extinction spectrum of the nanoprisms is also sensitive to the thickness of the particles and to "snipping" (truncation of the tips of the triangles). The long wavelength in-plane dipole resonance shifts to the red when the edge length is increased or the thickness is decreased, while snipping moves the plasmon resonance to the blue. The quadrupole plasmon mode is less sensitive to size or snipping, as the dominant induced polarization for this mode is localized to the central portion of the triangle, thereby avoiding the tip regions. 

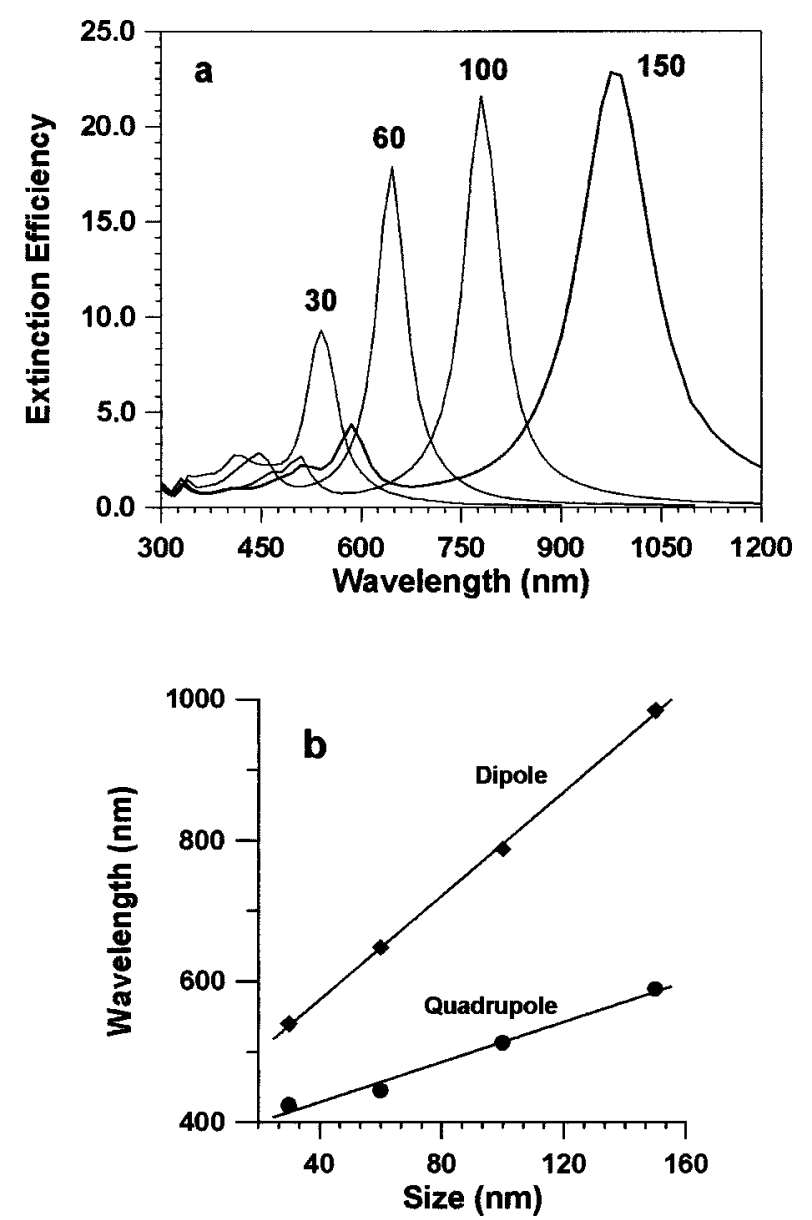

Fig. 1. (a) DDA simulations of the extinction efficiency of triangular nanoprisms. From left to right, the sizes of prisms are 30,60,100, and $150 \mathrm{~nm}$. The thickness of all prisms is $10 \mathrm{~nm}$. For all prisms, $15 \%$ of the tips have been truncated (snipped) to mimic the average behavior found in the experiments. (b) Plasmon wavelength as a function of triangle edge length.

\section{Ag Nanodisks}

The synthesis of $\mathrm{Ag}$ nanodisks involves a distinctly different approach [20] from the triangles that is schematically shown in Fig. 2. Here the reduction is done under surfactant-free conditions, and polystyrene meso-spheres are used to template the particle growth. Under these conditions, the $\mathrm{Ag}$ ions can electrostatically bind to the surface of the meso-spheres near to carboxyl groups. Upon reduction, the adsorbed $\mathrm{Ag}$ ions presumably serve to nucleate metal particle formation. Once nucleation is initiated the meso-spheres may block growth in one direction, effectively constraining growth to occur parallel to the PS surface. A transmission electron micrograph (Fig. 3a) of a fresh sample shows individual and aggregated polystyrene particles inhomogeneously covered by silver disks that

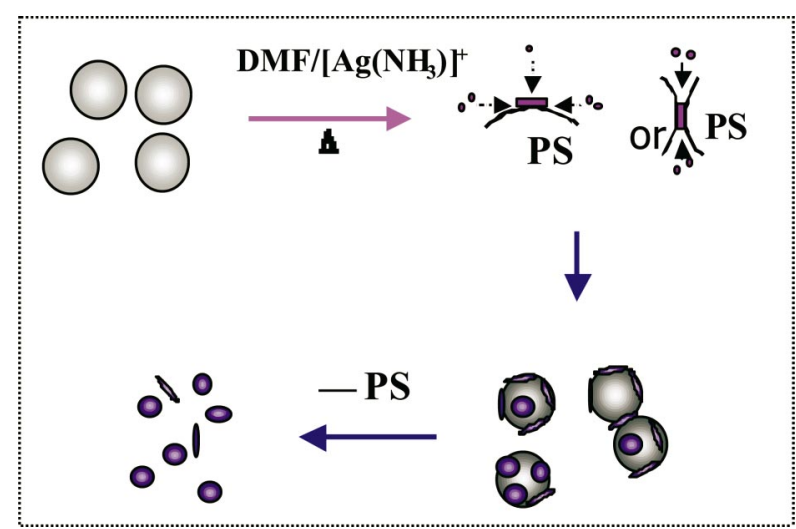

Fig. 2. Schematic procedure for the anisotropic growth of $\mathrm{Ag}$ nanodisks on the surface of polystyrene micro-spheres.

are randomly oriented on the particle surfaces. The disk shape was verified by tilting the TEM sample plane from $-30^{\circ}$ through $0^{\circ}$ to $+30^{\circ}$ (see Fig. $3 \mathrm{~b}$ ), revealing the threedimensional structure of the nanoparticles. At this stage almost no Ag particles unattached to polystyrene are seen. The presence of edge-oriented disks, appearing as rod-like shapes in the TEM images, allows measurement of their thickness $(9.0 \pm 1.0 \mathrm{~nm})$. The average disk diameter is $36 \pm 8 \mathrm{~nm}$. The available microscopy (see Fig. 3a) also suggests that anisotropic growth can occur between PS meso-spheres, leading to aggregation of the templating units.

The extinction spectrum, as shown in Fig. 4, exhibits surface plasmon resonances at $\lambda_{\max }=340 \mathrm{~nm}$ (weak) and $574 \mathrm{~nm}$ (strong), with other peaks between 430 and $470 \mathrm{~nm}$. To gain insight into the optical properties, we calculated the extinction spectrum of the Ag nanodisks considering its dimensions to given by TEM (see Fig. 4, dashed line). For the Ag disk, the induced polarizations lead to three bands that qualitatively match the experimental results shown in Fig. 4. Analysis of the induced field shows that the $574 \mathrm{~nm}$ peak is the in-plane dipole plasmon resonance, the $340 \mathrm{~nm}$ peak is the out-of-plane quadrupole resonance, and the weak $430 \mathrm{~nm}$ peak is the out-of-plane dipole resonance. Only the $574 \mathrm{~nm}$ peak is found to be very sensitive to the disk thickness and dimensions. These results are analogous to what was found for the triangular prisms in Fig. 1 (see the $30 \mathrm{~nm}$ result), although with different peak wavelengths, intensities and size sensitivities.

\section{Au Multipods}

Aqueous Au nanoparticles are usually prepared using citrate, $\mathrm{NaBH}_{4}$, and surprisingly hydrogen peroxide as reducing agents. The reduction rate with citrate or hydrogen 


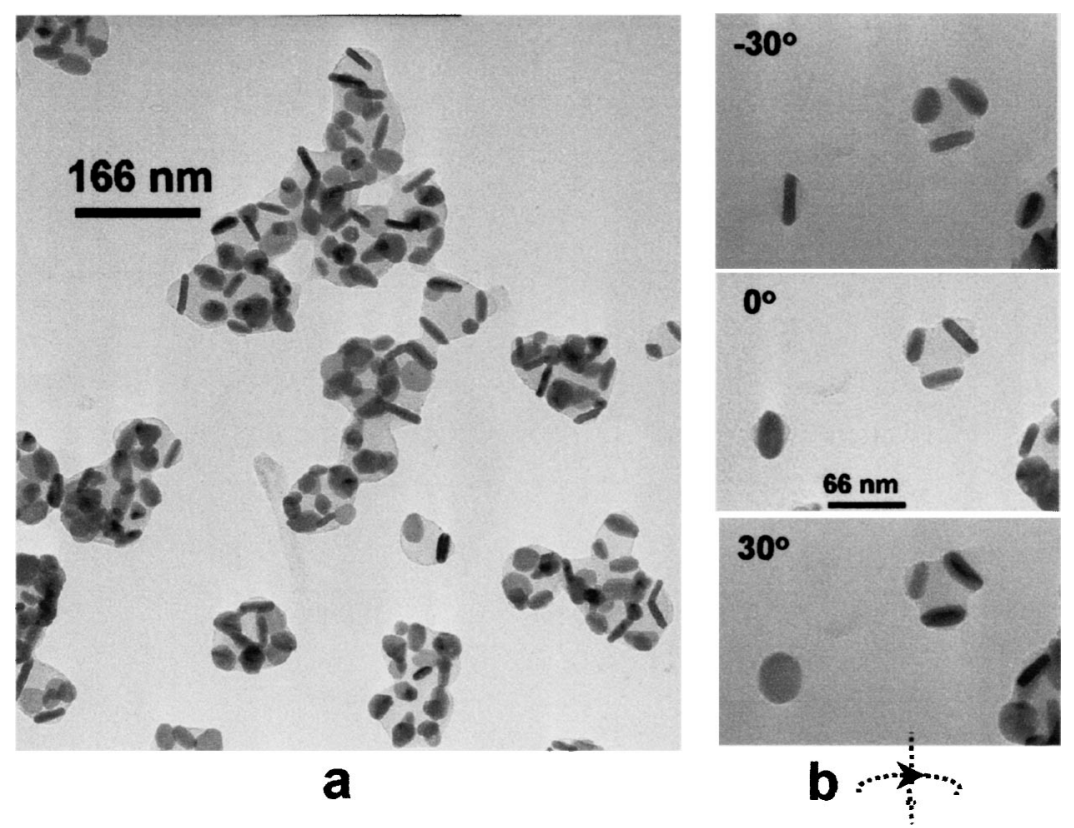

Fig. 3. (a) TEM image of a fresh Ag nanodisk/PS sample. (b) TEM images of Ag/PS, tilting the sample plane from $-30^{\circ}$ through $0^{\circ}$ to $+30^{\circ}$.

peroxide is very slow at room temperature and much faster at elevated temperature [60]. To our surprise, the reaction at room temperature is substantially accelerated when both are employed, with the formation of blue Au colloids taking place within several minutes.

As shown in Fig. 5, the gold nanoparticles from this synthesis have structurally unprecedented branched structures, with at least one well-defined tip on over $90 \%$ of the

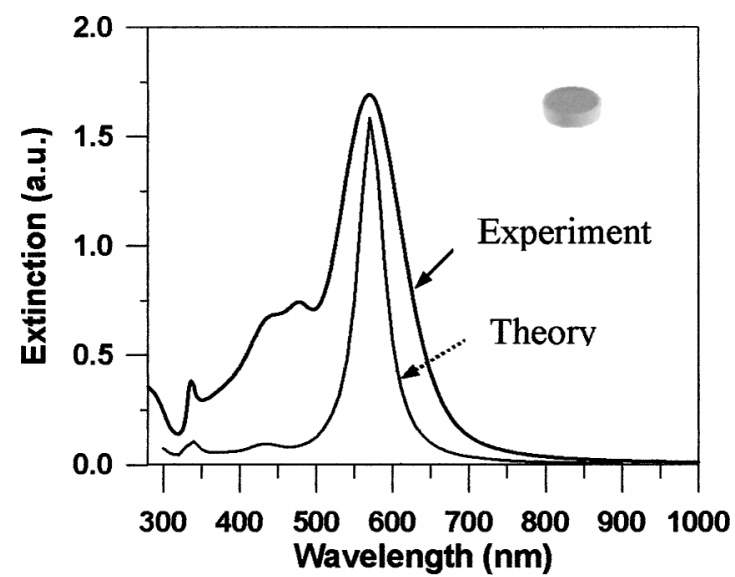

Fig. 4. Solid line is the UV-Vis spectrum of Ag nanodisk/PS in DMF. Dashed line is the DDA simulation of the orientation averaged extinction efficiency spectrum of a Ag disc (single size as described in the text) in DMF.
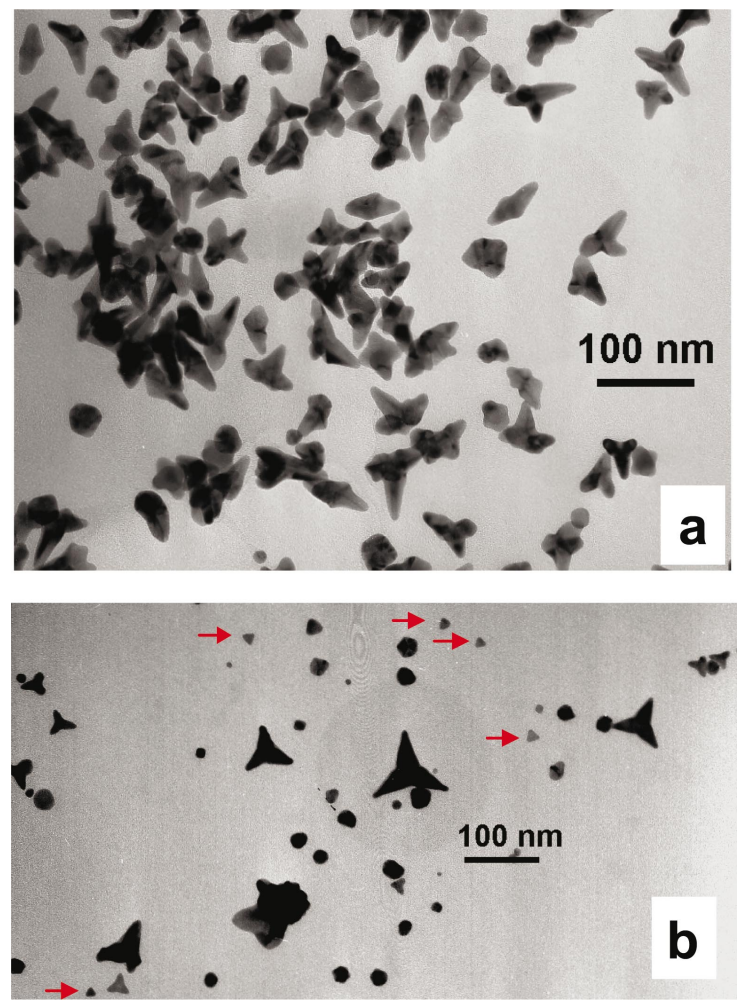

Fig. 5. (a) TEM images of branched gold nanocrystals. (b) TEM image of gold nanocrystals that were synthesized without BSPP during the initial particle growth. The arrows point out the presence of tiny triangular prisms that are discussed in the text. 
total particle population. Three-tipped nanoparticles are seen to be in the majority, comprising over 50\%. It's worth mentioning that the sharp curvature of each tip is different from the "multi-pods" that have been observed recently in several systems [29-32]. As discussed briefly below, this points to an unusual particle growth mechanism, differing from those for the triangular $\mathrm{Ag}$ prisms mentioned above that require a photodriven synthesis [4,28]. Note that room light illumination has no effect on the Au particle synthesis.

To date, the standard explanation for anisotropic particle growth in liquid media assumes that appropriate capping reagents kinetically control the growth rate of various facets of a seed particle [17,31]. Alivisatos and co-workers have recently demonstrated an extraordinary approach to CdSe nanorods and branched CdTe tetra-pods based on this concept [30]. In our experiments, synthesis without BSPP during initial growth produces "three-tipped" $\mathrm{Au}$ nanocrystals (Fig. 5b), indicating that the role of BSPP is not essential for formation of the branched structures. In addition, tiny triangular prisms (less than $10 \mathrm{~nm}$ edge length) are found in the same batch. We speculate that the tiny triangular prisms function as early-stage nuclei for "three-tipped" particle formation and growth. To produce "three-tipped" particles starting from triangular prisms, the growth has to be fastest perpendicular to the edge of the triangle, i.e., parallel to the $\{200\}$ direction and perpendicular to the $\{111\}$ direction. Although BSPP is not involved in the production of the symmetrical three-tipped particles in Fig. 5b, it is very important in producing asymmetrical branched structures (Fig. 5a), presumably by altering the growth rates of certain crystal facets of the seeds.

In contrast to the characteristic red color of spherical Au nanoparticles, the colloidal solution of the branched $\mathrm{Au}$ nanocrystals is blue. The measured extinction spectrum is shown in Fig. 6, and it shows a plasmon resonance peak that is red-shifted from ca. $500-530 \mathrm{~nm}$ for spheres to $690 \mathrm{~nm}$ for the branched Au particles. From a practical standpoint, the shape dependent optical properties of reactive gold colloids represent convenient experimental reporters in studies of crystal growth.

To understand the measured optical features in Fig. 6, we have solved Maxwell's equations for light interacting with a $14 \mathrm{~nm}$ thick "three-tipped" gold nanocrystal featuring $48 \mathrm{~nm}$ tip-to-tip distances and $4 \mathrm{~nm}$ snipping (meaning that, as mentioned earlier, a $4 \mathrm{~nm}$ region at the tips is removed in order to mimic the expected annealing of the sharpest features). As shown in Fig. 6, the calculated orientationally averaged spectrum for the three-tip particle shows two absorption bands at $700 \mathrm{~nm}$ (strong) and $540 \mathrm{~nm}$ (weak). Examination of the induced polarizations associated with these spectral features indi-

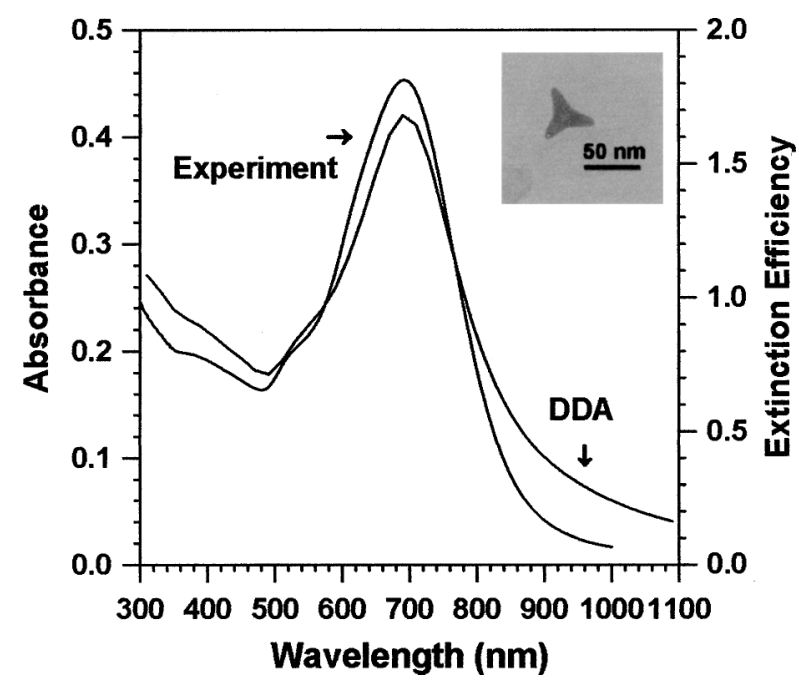

Fig. 6. UV-visible spectra of 3-tip branched gold nanocrystals (tip-tip distance $=48 \mathrm{~nm}$, and thickness $=14 \mathrm{~nm}$, snip $=4 \mathrm{~nm}$ ). The line labelled DDA represents the calculated extinction spectrum using the DDA method.

cates that the $700 \mathrm{~nm}$ peak is the in-plane dipole plasmon resonance and the $540 \mathrm{~nm}$ peak is the out-of-plane quadrupole resonance. We find that the calculated lineshape agrees qualitatively with the experimental spectrum, and quantitatively with respect to the wavelength of the plasmon maximum. Further calculations indicate that the plasmon bands of the three-tipped Au nanocrystals are very sensitive to the length and the sharpness of the tips, but less sensitive to the thickness and overall size of the particles.

As shown in Fig. 6, we observed relatively narrow plasmon absorption lineshapes from the branched $\mathrm{Au}$ nanocrystals. However the Au nanocrystals are not uniform (see Fig. 5), so it is surprising that the measured plasmon band is about the same width as the calculated one. To study this, we have performed calculations for $\mathrm{Au}$ nanocrystals that have three tips, two tips and even one tip. We find that these nanostructures have a similar extinction spectrum to the symmetrical particle, with plasmon resonances that are centered near $695 \mathrm{~nm}$. (One exception is the one-tipped particles that are blue-shifted to $674 \mathrm{~nm}$ ). Based these results, it is clear that in-plane dipole excitation of the particle tips dominates the optical properties of these particles, and (fortunately) the heterogeneous shape distribution has a rather small effect on the spectrum.

\section{Au Nanoshells}

Metallic nanoshells are one of the most interesting and possibly useful of the recently developed nanoparticle structures [35-40]. These particles usually consist of a thin 

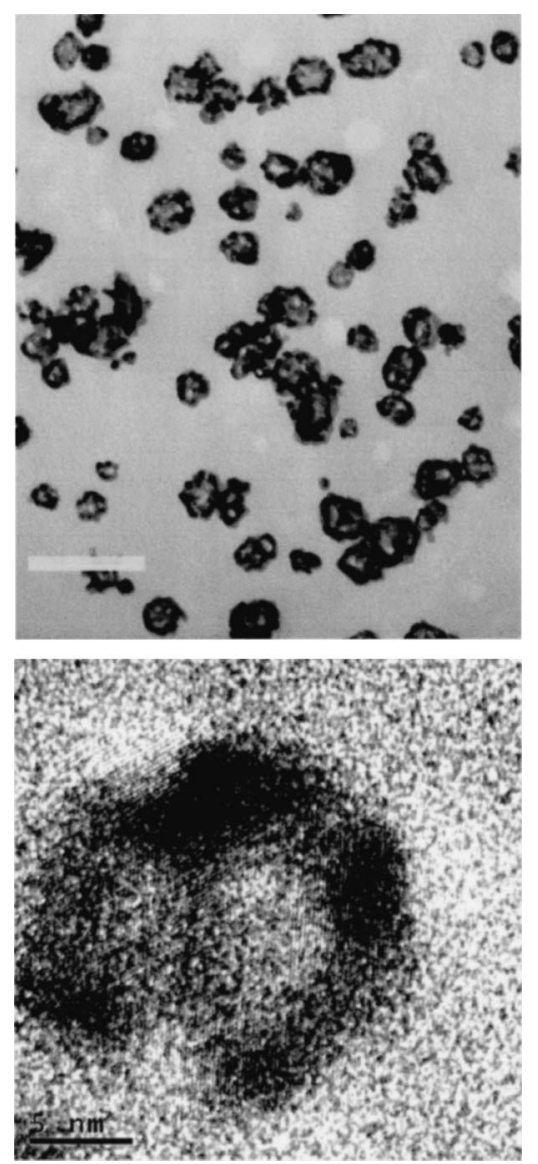

Fig. 7. (a) TEM photograph of Au nanoshells. The scale bar is $100 \mathrm{~nm}$. (b) High resolution TEM photograph of an individual Au nanoshell.

metallic ( $\mathrm{Au}$ or $\mathrm{Ag}$ ) shell around a dielectric core (such as $\mathrm{SiO}_{2}$ or polystyrene) $[37,39]$. Recently, silver nanoparticles including spheres [40,44,56], rods, cubes [33], and triangular prisms [48] have been etched with $\mathrm{HAuCl}_{4}$ to generate hollow particles. Sun and Xia generated seamless Au nanoshells having hollow interiors by combining the replacement reaction with annealing [44]. The resulting nanoshells have plasmon resonances that are strongly red-shifted compared to solid gold spheres, with a wavelength that is tunable depending on the ratio of the shell thickness to its diameter.

In our experiments [56], we synthesize Au nanoshells using a similar approach to that of Sun and Xia [44], but without annealing at elevated temperature. As shown in Fig. 7, the Au nanoshells have a broad size distribution ranging from 20 to $40 \mathrm{~nm}$. The size distribution of the nanoshells matches that of the Ag nanoparticle templates. An important feature of these particles is the presence of pinhole (several $\mathrm{nm}$ ) structures, which may allow both $\mathrm{HAuCl}_{4}$ and $\mathrm{Ag}^{+}$to continuously diffuse across the shell until the entire Ag template has been etched. Since a TEM image is a 2-dimensioned view of the nanoparticles, the white spots on the nanoshells represent the smallest size of holes, as this is where the electron beams go through holes in both the top and bottom of the nanoshells. The HRTEM image (Fig. 7b) of an individual Au nanoshell shows that the shell thickness for this particle is about $3.0 \mathrm{~nm}$.

It has been demonstrated both experimentally and theoretically that nanoshells exhibit interesting extinction spectra that are strongly dependent on size and shell thickness [35,37-39]. Sometimes these nanoshells have pinhole-like defects. However, it is not known how the optical properties of the nanoshells depend on the pinhole structures. In order to provide insight into this, we used Mie theory [61] and the DDA method to calculate the extinction spectrum of the Au nanoshells.

The extinction spectrum of the initial silver particles shows a narrow band at $400 \mathrm{~nm}$, however the Au shell spectrum shows a broad maximum at $736 \mathrm{~nm}$. Figure 8 a shows Mie theory results for perfect shells, demonstrating the well-known result that the extinction spectrum is very sensitive to the thickness, with the plasmon resonance shifting red when thickness is decreased. Note that the dielectric constant in the DDA calculations is corrected for finite electron mean free path effects that are determined by the shell thickness. Similar calculations performed without this correction yield extinction spectra with sharper and more intense features. These results are similar to results for Au nanoshells with dielectric cores that were reported by Halas et al. [39], and for hollow nanoshells reported by Xia et al. [44].

Figure $8 \mathrm{~b}$ shows that the DDA results for the perfect shells are very similar to the Mie theory results (i.e., exact electrodynamics) for the same size (Fig. 8b), thus demonstrating that the DDA method is capable of describing the shell electrodynamics adequately. Further DDA calculations show that pinholes ( $2-5 \mathrm{~nm}$ in diameter) do not affect the extinction spectra of the nanoshells. Thus except for inhomogeneity, the calculated perfect nanoshell spectrum for nanoshells that are $36 \mathrm{~nm}$ in diameter and $2.5 \mathrm{~nm}$ in thickness is sufficient to explain the experimental observations. This result is different from what was concluded in the nanocup studies of Charnay et al. [62], who used FDTD calculations to show that cup structures $(50 \mathrm{~nm}$ hole in a $50 \mathrm{~nm}$ radius shell whose thickness is $25 \mathrm{~nm}$ ) produce plasmon resonances that are strongly shifted from the perfect shell resonances. In this case the influence of the hole on the shell spectrum is significant, as the area of the hole is comparable to the projected area of the shell. This contrasts with what we considered here, where our pinholes are only a few percent of the size of the shell. 

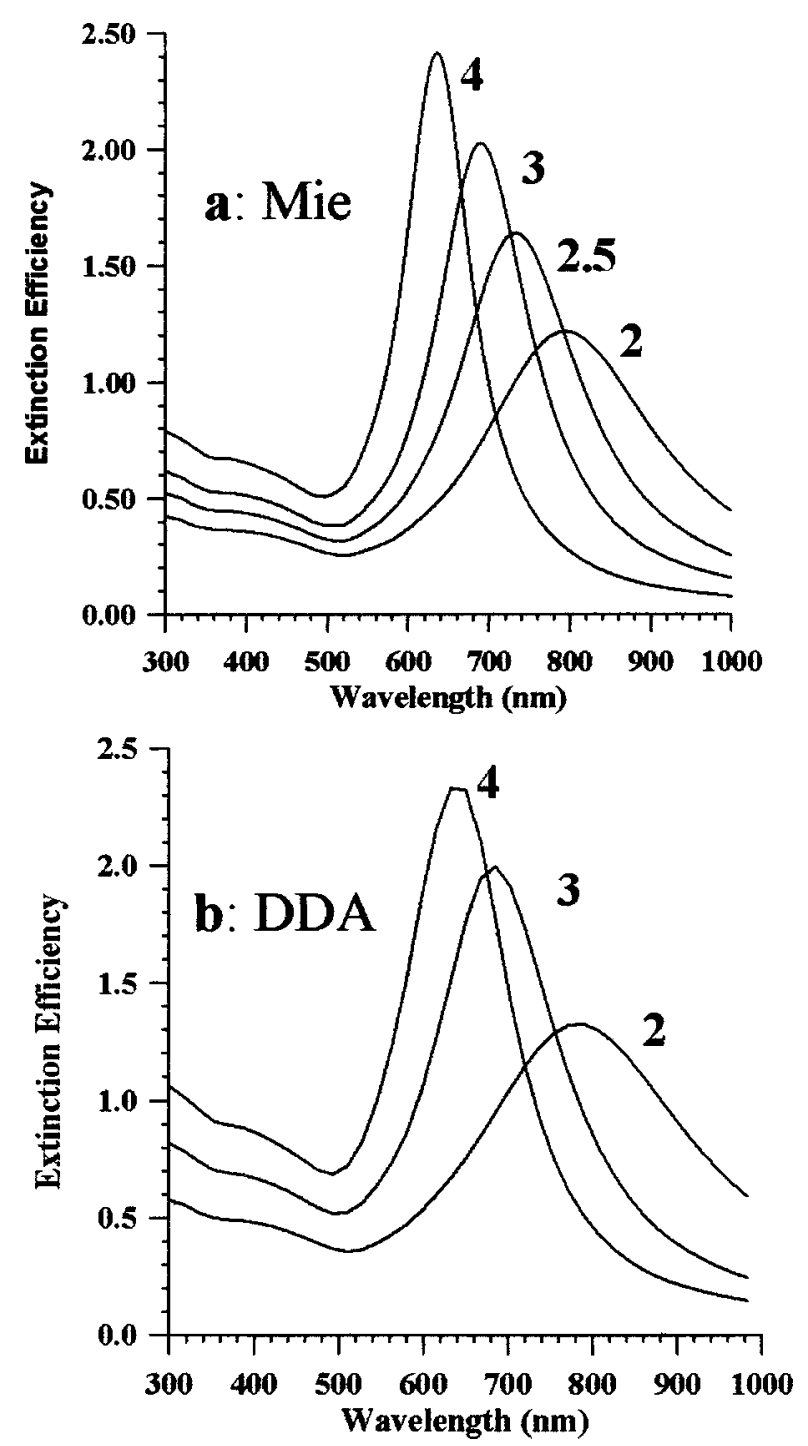

Fig. 8. (a) Mie theory extinction spectra of Au nanoshells (18 $\mathrm{nm}$ in radii) with varying shell thickness ranging from 2 to $4 \mathrm{~nm}$. (b) DDA extinction spectra of analogous Au nanoshells (18 nm in radii) with varying shell thickness.

\section{Local Electric Field Around Anisotropic Metal Nanoparticles}

Anisotropic metal nanoparticles may be attractive for applications that use surface enhanced Raman spectroscopy for chemical and biological sensing. SERS has been the subject of extensive studies, and it is generally agreed that an important contribution to the SERS enhancement comes from the electromagnetic (EM) field enhancements that occur near the particle surface as a result of plasmon excitation $[63,64]$. This E-field enhancement in turn leads to enhanced Raman scattering intensities. The electrodynamics of anisotropic metal nanoparticles

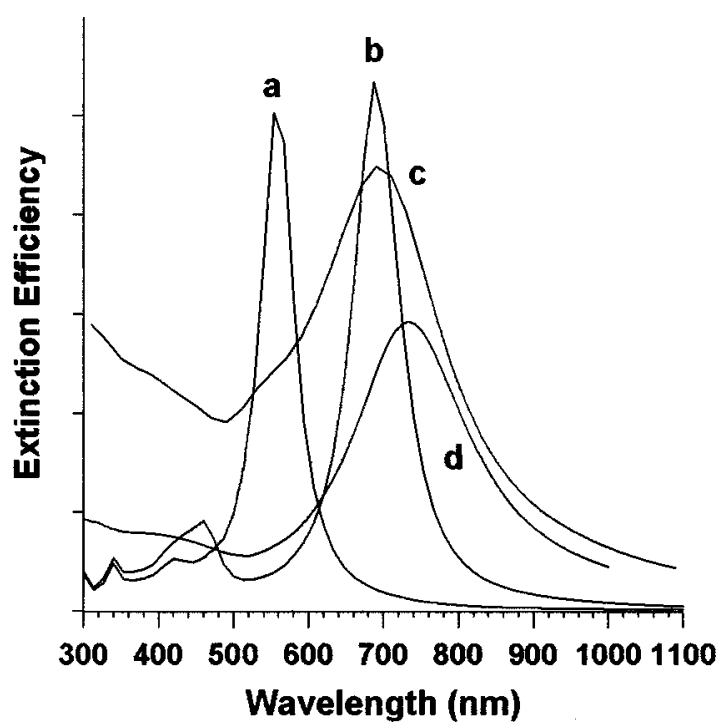

Fig. 9. The calculated extinction spectra of (a) silver nanodisks ( size $=40$ $\mathrm{nm}$, thickness $=9 \mathrm{~nm}$ ); (b) silver triangular nanoprisms without snipping ( size $=60 \mathrm{~nm}$, thickness $=12 \mathrm{~nm}$; (c) 3-tip branched gold nanocrystals (tip-tip distance $=48 \mathrm{~nm}$, and thickness $=14 \mathrm{~nm}$, snip $=4 \mathrm{~nm}$ ); and (d) gold nanoshells (radii $=18 \mathrm{~nm}$, shell thickness $=3 \mathrm{~nm}$ ).

has thus been of interest to estimates of the SERS enhancement factor.

Figure 9 shows the calculated extinction spectra of metal nanoparticles for several of the shapes that we have considered above, as well as other important shapes. The triangular prism has a $60 \mathrm{~nm}$ edge dimension with a snip of $2 \mathrm{~nm}$ and with a thickness of $12 \mathrm{~nm}$. 36,480 dipoles are used in the calculation. The dimensions of the $\mathrm{Ag}$ nanodisks, Au nanoshells, and branched Au nanocrystals are the same as discussed in Figs. 4, 6, and 8. Note that the Au spectra are consistently broader than the Ag spectra. This leads to more distinct multipole resonance structure for Ag, but the wavelengths of the dipolar plasmon resonances for the $\mathrm{Au}$ and $\mathrm{Ag}$ particles are about the same.

DDA methods can easily be modified to estimate the electromagnetic field (E-field) near the particle surface. Figure 10 shows the E-field enhancement for different shaped nanoparticles, plotted as contours of $\left|\mathbf{E}^{2}\right|$. The E-field contours for Au nanospheres are similar to that of the Ag nanospheres (as shown in Fig. 10a). The peak E-field of the Au nanospheres at $520 \mathrm{~nm}$ is about 36 times the applied field. As shown in Fig. 10, the anisotropic nanoparticles produce much stronger E-fields than that of the nanospheres, particularly for nanoparticles with sharp points such as triangular nanoprisms and branched nanocrystals. For nanoshells, the presence of hole defects does not have obvious influence 

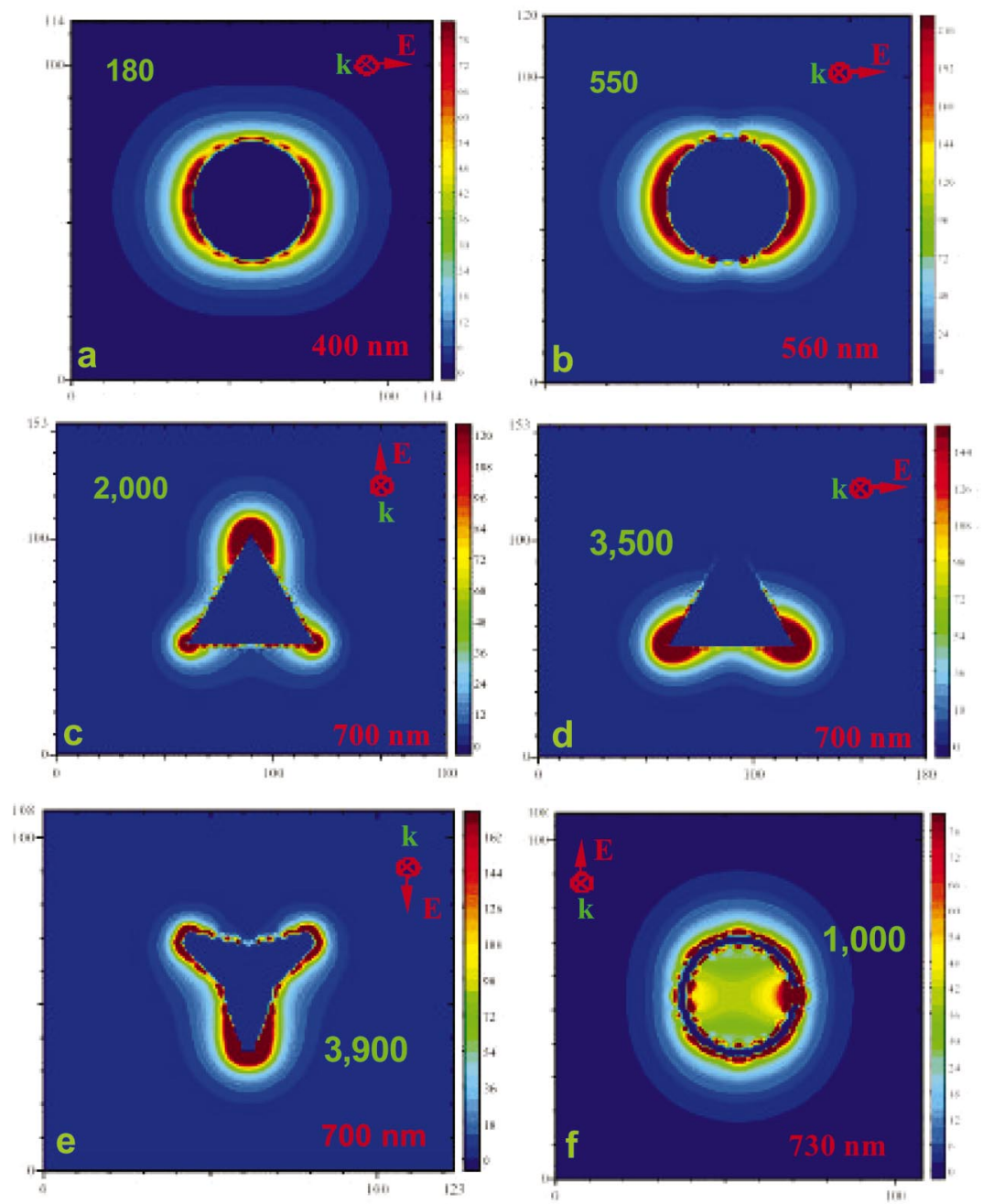

Fig. 10. E-field enhancement contours external to nanoparticles with different shapes, including (a) $\mathrm{Ag}$ nanospheres (radii $=19 \mathrm{~nm}$ ); (b) $\mathrm{Ag}$ nanodisks as described in Fig. 9; (c) and (d) $\mathrm{Ag}$ triangular nanoprisms as described in Fig. 9; (e) three-tipped Au nanocrystals as described in Fig. 9; (f) Au nanoshells as described in Fig. 9, but with a hole defect (hole size $=3 \mathrm{~nm}$ ).

on the extinction spectrum, but does create "hot" sites where the E-field enhancements are comparable to those of the triangular prisms, branched nanoparticles, and nanorods. The ability to support large E-field enhancements makes these anisotropic nanostructures very attractive for applications in SERS and environmental sensing.

\section{SUMMARY AND FUTURE WORK}

Using wet-chemical methods, we have demonstrated that it is possible to synthesize several anisotropic metal nanoparticles, including silver nanodisks, branched gold nanocrystals, and gold nanoshells. In comparison to silver and gold nanospheres, these new materials show intriguing optical properties with tunable surface plasmon bands that depend on the shapes of the particles.

For silver triangular nanoprisms, the DDA method provides an accurate description of the experimental results, indicating that DDA is a powerful tool to study the optical properties of metal nanoparticles with arbitrary shapes. The calculated results show that the surface plasmon band of triangular nanoprisms is sensitive to the edge length, to thickness, and to snipping. The long wavelength in-plane dipole resonance shifts to the red when the edge length is increased or the thickness is decreased, while snipping always moves the plasmon resonance to the blue. 
The silver nanodisk synthesis provides new ideas for the growth of anisotropic nanoparticles using submicrometer sized templates in liquid media. The silver nanodisks also exhibit interesting optical features that can be simulated using the DDA calculations. In comparison to silver nanospheres, the surface plasmon band strongly shifts to the red, with the wavelength depending on thickness and disk diameter. In many respects the optical properties of the silver disks are similar to that of smaller silver triangles, however the disks do not require a photodriven synthesis.

We also demonstrated that gold nanoparticles may be synthesized that have branched structures, especially 3-tipped particles. This is a great addition to the family of anisotropic nanoparticles. Gold triangular prisms were generated half a century ago, but in low yield, without distinctive tips, and in the micron-size range. For the first time, we developed a synthesis in which anisotropic particles with one, two or three points are produced in high overall yields. In addition, the extinction spectrum of these gold nanoparticles is surprisingly homogeneous, with a plasmon wavelength that is red shifted by more than 130 $\mathrm{nm}$ from that of gold nanospheres. The DDA calculations predict that the relatively narrow absorption is dominated by the distinctive tips, which is why the spectrum is not strongly dependent on the number of tips.

Nanoshells provide another way to adjust the surface plasmon band, here giving a plasmon wavelength that is strongly dependent on shell thickness and particle diameter. Our synthesis produces gold nanoshells with nanohole structures as indicated by TEM measurements. The theoretical calculations suggest that these pinhole structures (only few nanometers in diameter) do not affect the plasmon resonances of gold nanoshells.

SERS has been the subject of extensive studies, and it is generally agreed that an important contribution to the SERS enhancement comes from enhanced electromagnetic (EM) fields that are associated with plasmon excitation. Using the DDA method, one can estimate the SERS enhancement factor by calculating the enhanced Efield near the particle surface. In comparison to gold and silver nanospheres, the anisotropic nanoparticles can produce enormous E-field enhancements upon plasmon excitation, where the peak E-fields typically occur close to the sharp points for triangular prisms and branched nanoparticles. The pinhole structures of the nanoshells also create hot spots, leading to larger E-field enhancements. These results suggest that the current generation of anisotropic nanoparticles is not only of fundamental interest, but is also very attractive for applications in SERS, second harmonic Rayleigh scattering, and biological and chemical sensors.
The study of anisotropic nanoparticles is an emerging and rapidly developing research field. Although some success has been achieved in generating anisotropic nanoparticles, there are still great challenges, including (1) developing reliable approaches to different materials; (2) understanding the shape-guiding mechanisms, and therefore the possibilities for systemic manipulation of the final structures; (3) developing new theoretical methods to better describe the intriguing optical and electronic properties. As described above, these novel materials have great potential for advanced applications. However there is always room for improvement, so the self-assembly and stabilization of these materials with well-defined dimensions and orientations on surfaces will be an attractive direction for further research.

\section{ACKNOWLEDGMENT}

We gratefully acknowledge support from the National Science Foundation through the Materials Research Center (MRSEC) and the Air Force Office of Scientific Research MURI program (F49620-02-1-0381).

\section{REFERENCES}

1. A. P. Alivisatos (1996). Perspectives on the physical chemistry of semiconductor nanocrystals. J. Phys. Chem. 100, 13226-13239.

2. B. O. Dabbousi, J. Rodriguez-Viejo, F. V. Mikulec, J. R. Heine, H. Mattoussi, R. Ober, K. F. Jensen, and M. G. Bawendi (1997). J. Phys. Chem. B 101, 9463-9475.

3. M. A. Hines and P. Guyot-Sionnest (1996). J. Phys. Chem. 100, 468-471.

4. R. Jin, Y. Cao, C. A. Mirkin, K. L. Kelly, G. C. Schatz, and J. G. Zheng (2001). Science 294, 1901-1903.

5. K. L. Kelly, E. Coronado, L. L. Zhao, and G. C. Schatz (2003). J. Phys. Chem. B 107, 668-677.

6. S. Link and M. A. El-Sayed (1999). J. Phys. Chem. B 103, 84108426.

7. C. B. Murray, D. J. Norris, and M. G. Bawendi (1993). J. Am. Chem. Soc. 115, 8706-8715.

8. X. Peng, M. C. Schlamp, A. V. Kadavanich, and A. P. Alivisatos (1997). J. Am. Chem. Soc. 119, 7019-7029.

9. M. Gao, S. Kirstein, H. Möhwald, A. L. Rogach, A. Kornowski, A. Eychmuller, and H. Weller (1998). J. Phys. Chem. B 102, 8360-8363.

10. A. C. Templeton, W. P. Wuelfing, and R. W. Murray (2000). Acc. Chem. Res. 33, 27-36.

11. B. D. Busbee, S. O. Obare, and C. J. Murphy (2003). Adv. Mater. 15, $414-416$

12. S.-S. Chang, C.-W. Shih, C.-D. Chen, W.-C. Lai, and C. R. C. Wang (1999). Langmuir 15, 701-709.

13. A. Chemseddine and T. Moritz (1999). Eur. Inorg. Chem. 235-245.

14. N. R. Jana, L. Gearheart, and C. J. Murphy (2001). Chem. Commun., 617-618.

15. F. Kim, J. H. Song, and P. Yang (2002). J. Am. Chem. Soc. 124, 14316-14317.

16. Z. Tang, N. A. Kotov, and M. Giersig (2002). Science 297, 237-240.

17. Y. N. Xia, P. D. Yang, Y. G. Sun, Y. Y. Wu, B. Mayers, B. Gates, Y. D. Yin, F. Kim, and Y. Q. Yan (2003). Adv. Mater. 15, 353389. 
18. Y.-Y. Yu, S.-S. Chang, C.-L. Lee, and C. R. C. Wang (1997). J. Phys. Chem. B 101, 6661-6664.

19. S. Chen, Z. Fan, and D. L. Carroll (2002). J. Phys. Chem. B 106, 10777-10781.

20. E. Hao, K. L. Kelly, J. T. Hupp, and G. C. Schatz (2002). J. Am. Chem. Soc. 124, 15182-15183.

21. M. Maillard, S. Giorgio, and M.-P. Pileni (2002). Adv. Mater. 14, 1084-1086.

22. V. F. Puntes, D. Zanchet, C. K. Erdonmez, and A. P. Alivisatos (2002). J. Am. Chem. Soc. 124, 12874-12880.

23. M. Maillard, P. Huang, and L. Brus (2003). Nano Lett. 3, 1611-1615.

24. S. Chen and D. L. Carroll (2002). Nano Lett. 2, 1003-1007.

25. I. Pastoriza-Santos and L. M. Liz-Marzán (2002). Nano Lett. 2, $903-$ 905.

26. Y. Sun, B. Mayers, and Y. Xia (2003). Nano Lett. 3, 675-679.

27. J. Yang and J. H. Fendler (1995). J. Phys. Chem 99, 5505-5511.

28. R. Jin, Y. Cao, E. Hao, G. Metraux, G. C. Schatz, and C. A. Mirkin (2003). Nature 425, 487-490.

29. S.-M. Lee, Y.-W. Jun, S. N. Cho, and J. Cheon (2002). J. Am. Chem. Soc. 124, 11244-11245; S. Chen, Z. L. Wang, J. Ballato, S. H. Foulger, and D. L. Carroll (2003). J. Am. Chem. Soc. 125, 1618616187.

30. L. Manna, D. J. Milliron, A. Meisel, E. C. Scher, and A. P. Alivisatos (2003). Nature Mater. 2, 382-385.

31. L. Manna, E. C. Scher, and A. P. Alivisatos (2000). J. Am. Chem. Soc. 122, 12700-12706.

32. E. Hao, R. C. Bailey, G. C. Schatz, J. T. Hupp, and S. Li (2004). Nano Lett. 4, 327-330.

33. Y. Sun and Y. Xia (2002). Science 298, 2139-2141.

34. T. S. Ahmadi, Z. L. Wang, T. C. Green, A. Henglein, and M. A. El-Sayed (1996). Science 272, 1924-1926.

35. S. J. Oldenburg, J. B. Jackson, S. L. Westcott, and N. J. Halas (1999). Appl. Phys. Lett. 75, 2897-2899.

36. J. B. Jackson and N. J. Halas (2001). J. Phys. Chem. B 105, $2743-$ 2746.

37. C. Graf and A. v. Blaaderen (2002). Langmuir 18, 524-534.

38. E. Pordan and P. Nordlander (2003). Nano Lett. 3, 543-547.

39. S. J. Oldenburg, R. D. Averitt, S. L. Westcott, and N. J. Halas (1998). Chem. Phys. Lett. 288, 243-247.

40. Y. Jin and S. Dong (2003). J. Phys. Chem. B 107, 12902-12905.

41. M. B. Mohamed, V. Volkov, S. Link, and M. A. El-Sayed (2000). Chem. Phys. Lett. 317, 517-523.

42. X. Peng, L. Manna, W. Yang, J. Wickham, E. Scher, A. Kadavanich, and A. P. Alivisatos (2000). Nature 404, 59-61.
43. W. U. Huynh, J. J. Dittmer, and A. P. Alivisatos (2002). Science 295, $2425-2427$.

44. Y. Sun and Y. Xia (2002). Anal. Chem. 74, 5297-5305.

45. B. Nikoobakht, J. Wang, and M. A. El-Sayed (2002). Chem. Phys. Lett. 366, 17-23.

46. E. Hao and G. C. Schatz (2004). J. Chem. Phys. 120, 357-366.

47. Y. A. Sun and Y. N. Xia (2003). Adv. Mater. 15, 695-699.

48. G. S. Metraux, Y. C. Cao, R. C. Jin, and C. A. Mirkin (2003). Nano Lett. 3, 519-522.

49. S. O. Obare, N. R. Jana, and C. J. Murphy (2001). Nano Lett. 1, 601-603.

50. B. T. Draine and P. J. Flateau. User Guide for the Discrete Dipole Approximation DDSCAT.6.0, http://arxiv.org/abs/astro-ph0309069, with modifications described in W. H. Yang, G. C. Schatz, and R. P. Van Duyne (1995). J. Chem. Phys. 103, 869-875.

51. T. Jensen, K. L. Kelly, A. Lazarides, and G. C. Schatz (1999). J. Cluster Sci. 10, 295-317.

52. K. L. Kelly, A. A. Lazarides, and G. C. Schatz (2001). Comput. Sci. Eng. 3, 67-73.

53. R. X. Bian, R. C. Dunn, X. S. Xie, and P. T. Leung (1995). Phys. Rev. Lett. 75, 4772-4775.

54. P. D. Cozzoli, A. Kornowski, and H. Weller (2003). J. Am. Chem. Soc. 125, 14539-14548.

55. S.-J. Park, S. Kim, S. Lee, Z. G. Khim, K. Char, and T. Hyeon (2000). J. Am. Chem. Soc. 122, 8581-8582.

56. E. Hao, S. Li, R. C. Bailey, G. C. Schatz, and J. T. Hupp (2004). J. Phys. Chem. B 108, 1224-1229.

57. K. L. Kelly, T. R. Jensen, A. A. Lazarides, and G. C. Schatz (2001). in D. Feldheim and C. Foss (Ed.), Metal Nanoparticles: Synthesis, Characterization and Applications, Marcel-Dekker, New York, pp. 89-118.

58. E. Coronado and G. C. Schatz (2003). J. Chem. Phys. 119, 39263934.

59. E. D. Palik (1985). Handbook of Optical Constants of Solids, Academic Press, New York.

60. W. O. Milligan and R. H. Morriss (1964). J. Am. Chem. Soc. 86, 3461-3467.

61. G. Mie (1908). Ann. Phys. 25, 377-445.

62. L. Charnary, A. Lee, S. Q. Man, C. E. Moran, C. Radloff, R. K. Bradley, and N. J. Halas (2003). J. Phys. Chem. B 107, 73277333.

63. G. C. Schatz (2001). J. Mol. Struc. (Theochem.) 573, 7380.

64. G. C. Schatz (1984). Acc. Chem. Res. 17, 370-376. 